\title{
Colonic Hemorrhage, CTCAE
}

National Cancer Institute

\section{Source}

National Cancer Institute. Colonic Hemorrhage, CTCAE. NCI Thesaurus. Code C143374.

A disorder characterized by bleeding from the colon. 